\title{
Effect of estrogen administration on middle cerebral arterial viscoelastic properties in aged female rats ${ }^{1}$
}

\author{
Zhigang Qin', Guijie Li' ${ }^{I I}$ Naijie Liu', Conghai Zhao', Hang Zhao ${ }^{I}$ \\ DOI: http://dx.doi.org/10.1590/S0102-865020160100000004 \\ 'Doctor, Department of Neurosurgery, Sino-Japanese Friendship Hospital, Jilin University, Changchun, China. Design of the study, manuscript writing, \\ critical revision. \\ "Master, Department of Otolaryngology, Head and Neck Surgery, Sino-Japanese Friendship Hospital, Jilin University, Changchun, China. Design of \\ the study.
}

\begin{abstract}
PURPOSE: To develop a model for studying cerebrovascular disease prevention in elderly women.

METHODS: Sixty 18-month-old Sprague Dawley (SD) rats were randomly divided into an estrogen administration group (EA, $\mathrm{n}=30$ ) and a non-administration group (NA, n=30); thirty 4-month-old SD rats were allocated to a control group. The EA group received estradiol benzoate starting on the 5th day of a 34-day breeding period, and the serum levels of estradiol (E2), estrogen receptor (ER), and malondialdehyde (MDA) were measured. The MCA of each group was then sampled for viscoelastic experiments.

RESULTS: The serum levels of E2 and MDA in the EA group showed significant differences compared to those in the control group $(p<0.05)$, while the difference in ER between the EA and control groups was not significant $(p>0.05)$. The decrease in MCA stress at $7,200 \mathrm{~s}$ and the increase in strain at 7,200 $\mathrm{s}$ in the EA group showed no significant differences compared to the control group ( $\mathrm{p}>0.05$ ).

CONCLUSION: Estradiol administration inhibited the formation of lipid peroxidation products and restored middle cerebral arterial viscoelasticity in aged female rats.
\end{abstract}

Key words: Aging. Estrogens. Middle Cerebral Artery. Female. Rats. 


\section{Introduction}

Numerous clinical studies have reported that the incidence of cardiovascular disease (CVD) in women begins to increase during menopause, indicating that estrogen deficit plays an important role in atherosclerosis and CVD. Estrogen replacement therapy used to treat menopausal symptoms reportedly decreased the relative risk of stroke, and that stroke-related mortality was also decreased ${ }^{1}$. Much research has evaluated the relationships between sex hormones, cerebral vessels, and neurological diseases ${ }^{2-5}$. Won et al. ${ }^{6}$ created a rat model of middle cerebral artery (MCA) occlusion, and found that estrogen administration (EA) could inhibit the overexpression of $\mathrm{Bcl}-2$ protein and activation of nuclear transcription factors in ischemic areas, thereby reducing apoptosis and inflammatory reactions. Momoi et al. ${ }^{7}$ resected the gonads of male and female rats, and then performed EA. They found that estrogen could induce the microvascular system of gonadectomized rats to release $\mathrm{NO}$, which was more obvious in female rats, and concluded that estrogen could regulate the release of NO, thus improving brain microvessel quality, maintaining cerebral blood flow, and exhibiting protective effects against cerebral ischemia. Only a few researchers have reported the effect of estrogen on intracranial arterial biomechanical characteristics. Ibrahim et al. ${ }^{8}$ studied myogenic regulatory function of the MCA in normal male and female WKY rats and those with spontaneous hypertensive stroke (WKY), and found that MCA in the normal female WKY group was not significantly increased to the extent of pressure expansion, while that in the Spontaneously Hypertensive Stroke Prone Rat (SH RSP) group was significantly increased; the vascular stiffness in the normal female WKY group was stronger than that in the male group, while that in the SH RSP group was significantly decreased compared to that in the male group.

One report discussed the effect of EA on MCA viscoelastic properties. Estradiol (E2) administration increased the elasticity, strength, and compliance of the MCA in aged rats ${ }^{9}$. Therefore, it could be inferred that changes in sex hormone levels might show a correlation with intracranial viscoelastic properties; if EA could increase estrogen levels in aged female rats, it might restore viscoelastic properties. The purpose of this paper was to use viscoelastic and biological indicators to evaluate the effects of EA in aged rats, thus providing a basis for EA in preventing cerebrovascular disease in elderly women.

\section{Methods}

The animal use protocol has been reviewed and approved by the Institutional Animal Care and Use Committee (IACUC) of Jilin University.

Sixty 18-month-old female Sprague Dawley (SD) rats (clean-level, body weight 290-299g) and 30 4-month-old SD rats (clean-level, body weight 291-300g) were obtained from Changchun High-tech Medical Laboratory Animal Center (license number SCXK (Ji) 2003-0004). The animals were bred at room temperature $\left(20 \pm 1^{\circ} \mathrm{C}\right)$, with air circulation, relative humidity $55-70 \%$, and a natural light cycle. The animals were caged with free food and water at Animal Biosafety Level 2, and feed was purchased from Shenyang Experimental animal feed factory, with the product standard code: DB-21741-93. This study was carried out in strict accordance with the recommendations in the Guide for the Care and Use of Laboratory Animals of the National Institutes of Health.

The 60 18-month-old female SD rats were randomly divided into an EA group ( $\mathrm{n}=30)$, and a non-administration (NA) group $(n=30)$; the 304 -month-old female SD rats were used as a control group.

\section{Estrogen administration (EA)}

The rats received intramuscular injections of estradiol benzoate $0.2 \mathrm{mg} / \mathrm{kg}$ starting on the 5 th day of breeding, once every other day. The control and NA groups received no treatment; all rats were bred for 34 days.

\section{Specimen sampling}

After 34 days of breeding, $6 \mathrm{ml}$ of orbital blood was sampled from 15 rats in each group, left to stand at room air for $15 \mathrm{~min}$, and centrifuged at low-speed $(2,000 \mathrm{rpm})$ for $20 \mathrm{~min}$; the supernatant was then preserved at $-40^{\circ} \mathrm{C}$ for later use; the rats then underwent craniotomy using an ASX-1 operating microscope (Shanghai Anxin Optics Manufacturing Co., China); the MCA was identified and resected with an S-5 sterile scalpel (Huaian Joint Yikang Medical Products, Xuyi, Jiangsu Province, China), Each group was sampled for 15 specimens, which were wrapped with saline-soaked cloth, and stored in a saline bath at $4^{\circ} \mathrm{C}$ for future use.

\section{Measurement of sample geometric size}

The geometric sizes of 15 MCA specimens from each group were measured using a CG-type reading microscope (Third Optical Instrument Factory, Changchun, Jilin, China). MCA 
specimens from the control, EA, and NA groups were all $10 \mathrm{~mm}$ long, with a diameter of 1.0-1.02 $\mathrm{mm}$.

\section{Determination of E2 and estrogen receptor (ER) concentration}

A microplate reader (LABsystems Co., Finland) and enzyme-linked immunosorbent assay (ELISA) kits were used according to instructions to determine serum concentrations of E2 and ER. The serum levels of E2 and ER were detected by radioimmunoassay (RIA), with instrument sensitivity set for $1.1 \mathrm{pg} / \mathrm{mL}$, intra-assay coefficient of variation $(\mathrm{CV}) \leq 10 \%$, and inter-assay $\mathrm{CV} \leq 10 \%$. The assay sensitivity $\mathrm{T}$ was $0.05 \mathrm{ng} / \mathrm{mL}$, the intra-assay $\mathrm{CV} \leq 10 \%$, and inter-assay $\mathrm{CV} \leq 10 \%$; the sample measurements used the double-tube parallel method; readings were obtained from the instrument, and corresponding concentrations were read from a standard curve.

\section{Determination of malondialdehyde (MDA)}

Analysis was performed according to the instructions of the MDA kit, and the thiobarbituric acid assay was used to detect serum MDA level. MDA in degradation products of lipid peroxide was condensed with thiobarbituric acid, thus forming a red product with maximum absorption peak at $532 \mathrm{~nm}$; absorption was then detected using a U3410 spectrophotometer (Hitachi, Japan); then, MDA content was calculated using the following formula (with homogenate concentration of $10 \%)$ : serum MDA $(\mathrm{nmol} / \mathrm{ml})=$ (absorbance of measurement tube - absorbance of blank tube) (absorbance of standard tube - absorbance of standard blank tube) $\times$ standard concentration $(\mathrm{IO} \mathrm{nmol} / \mathrm{ml}) \times$ dilution time of sample.

\section{Stress relaxation test}

Using a CSS4500 automatic electronic universal testing machine (Changchun Institute of test machine, Changchun, Jilin, China), each sample was preconditioned and treated as previously described $^{10,11}$. The tester was equipped with a thermostat that could be set from $-30^{\circ} \mathrm{C}$ to $250^{\circ} \mathrm{C}$; the ambient temperature of this experiment was set as $36.5 \pm 1.0^{\circ} \mathrm{C}$; each sample was clamped in a chuck on the test machine, and strain was applied to the sample at a rate of $30 \% / \mathrm{min}$; when the stress on each sample reached $16.0 \mathrm{kPa}$ (normal systolic blood pressure), the MCA strain in the normal group reached $39.0 \%$, the strain in the NA group reached $29.3 \%$, and the strain in the EA group reached $39.0 \%$; the strain values then remained constant. Stress decreased as time increased; saline was used to continuously moisten the samples during the experiment. After completion of $16.0 \mathrm{kPa}$ stress relaxation tests, the samples were subjected to $22.5 \mathrm{kPa}$ stress relaxation at a strain rate of $30 \% / \mathrm{min}$; when the stress reached $22.5 \mathrm{kPa}$, the MCA strain in the control group reached $48.2 \%$, the strain in the NA group reached $34.8 \%$, and the strain in the EA group reached $34.8 \%$; the strain values then remained constant.

\section{Creep test on the MCA}

The clamping method and preconditioning treatment for the creep test were the same as for the stress relaxation tests. Stress at an increasing rate of $0.5 \mathrm{MPa} / \mathrm{s}$ was applied to the 3 groups. When the stress in each group reached $16.0 \mathrm{kPa}$, the MCA strain of the control group reached $39.0 \%$ and the strain in the NA group reached $29.3 \%$; the stress then remained constant, and the strain was constantly increased as a function of time; after a preset test time was reached, the computer would automatically calculate the results. After the creep test with $16.0 \mathrm{kPa}$ was completed, the test was performed with $22.5 \mathrm{kPa}$ under constant stress, at an increasing stress rate of $0.5 \mathrm{MPa} / \mathrm{s}$; when the stress in each group reached $22.5 \mathrm{kPa}$, the MCA strain in the control group reached $48.2 \%$, the strain in the NA group reached $34.8 \%$, and the strain in the EA group reached $34.8 \%$, with the strain kept constant.

\section{Statistical analysis}

SPSS 11.0 software (SPSS, Chicago, IL, USA) was used for the paired t-test, with $\mathrm{p}<0.05$ considered statistically significant.

\section{Results}

\section{E2 and ER}

The serum levels of E2 and ER in each group are shown in Table 1. The serum values of E2 and ER in the EA group were greater than the levels in the NA group, and the difference was statistically significant $(\mathrm{p}<0.05)$.

TABLE 1 - Detection results of serum E2 and ER in each group.

\begin{tabular}{ccc}
\hline No & E2 & ER \\
\hline Control group & $54.8 \pm 1.3^{\mathrm{a}}$ & $19.6 \pm 1.2^{\mathrm{a}}$ \\
EI group & $55.8 \pm 1.4^{\mathrm{b}}$ & $21.2 \pm 1.3^{\mathrm{b}}$ \\
NI group & $42.6 \pm 2.9^{\mathrm{c}}$ & $16.8 \pm 1.7^{\mathrm{c}}$ \\
\hline
\end{tabular}

Note: The data were expressed as $\bar{x} \pm \mathrm{s}$, the intergroup comparison used the Sceffe method, ${ }^{a} \mathrm{p}<0.05$, vs the control group; ${ }^{\mathrm{b}} \mathrm{p}<0.05$, vs the EI group. ${ }^{\mathrm{c}} \mathrm{p}<0.05$, vs Aged rats-estrogen intervention group. 


\section{Serum MDA content}

The serum MDA level in the EA group was less than the level in the NA group, and the difference was statistically significant (Table 2, $\mathrm{p}<0.05$ ).

TABLE 2 - Detection of serum MDA content in each group $(\mathrm{mmol} / \mathrm{ml})$.

\begin{tabular}{cc}
\hline No & Mda $(\mathbf{m m o l} / \mathbf{m l})$ \\
\hline Control group & $7.08 \pm 0.26^{\mathrm{a}}$ \\
EI group & $6.96 \pm 0.31^{\mathrm{b}}$ \\
NI group & $8.52 \pm 0.41^{\mathrm{c}}$ \\
\hline
\end{tabular}

Note: The data were expressed as $\bar{x} \pm \mathrm{s}$, the intergroup comparison used the Sceffe method, ${ }^{a} \mathrm{p}<0.05$, vs the control group; ${ }^{\mathrm{b}} \mathrm{p}<0.05$, vs the EI group. ${ }^{\mathrm{c}} \mathrm{p}<0.05$, vs Aged rats-estrogen intervention group.

\section{MCA stress relaxation}

The relaxation curves with an initial stress of $16 \mathrm{kPa}$ on the MCA in different groups are shown in Figure 1, and those with stress of $22.5 \mathrm{kPa}$ are shown in Figure 2.

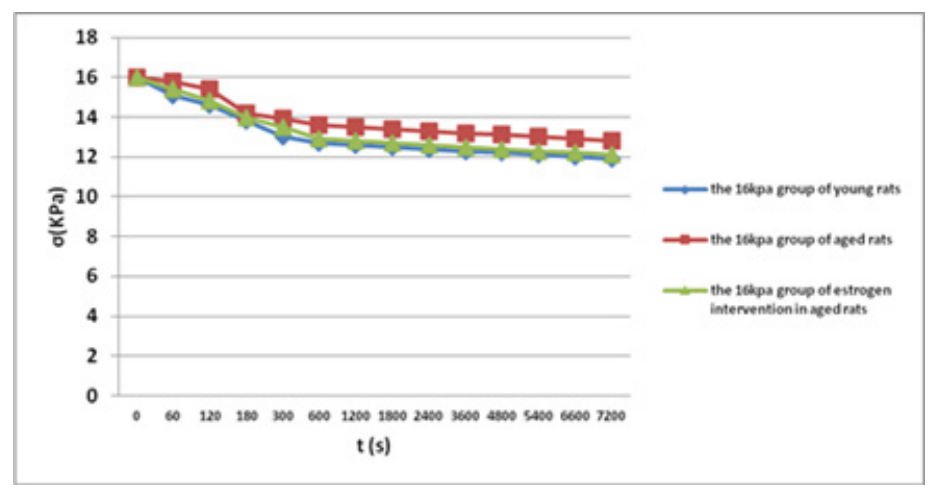

FIGURE 1 - Stress relaxation curves with initial stress of MCA of $16 \mathrm{Kpa}$ in different groups.

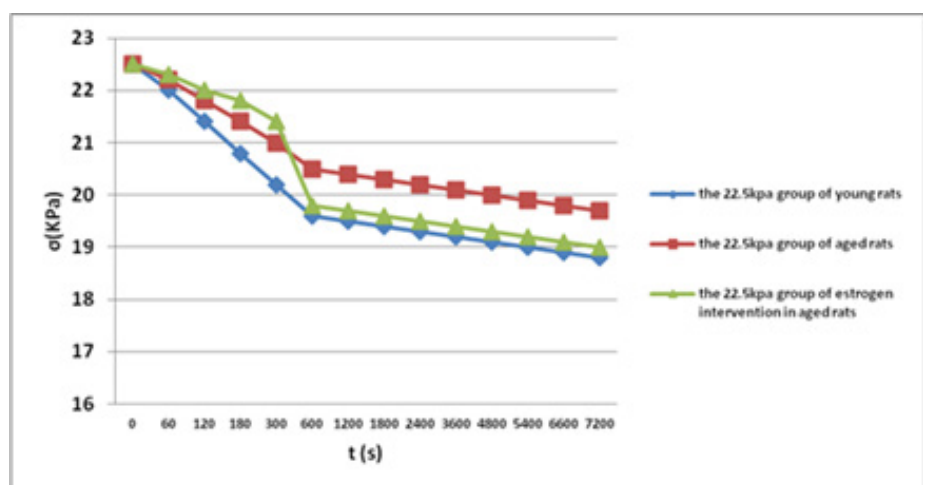

FIGURE 2 - Stress relaxation curves with initial stress of MCA of 22.5 Kpa in different groups.
When the initial stress on the MCA was set at $16 \mathrm{kPa}$, the 7.200 s stress on the MCA in the control group, the NA group, and the EA group was decreased in comparison with the values at $0 \mathrm{~s}$, by $4.1 \mathrm{kPa}, 3.2 \mathrm{kPa}$, and $3.9 \mathrm{kPa}$, respectively; the $7.200 \mathrm{~s}$ stress on the MCA in the EA group decreased more than in the NA group at $16 \mathrm{kPa}$ initial stress $(\mathrm{p}<0.05$; paired t-test). When the initial stress on the MCA was set at $22.5 \mathrm{kPa}$, the $7.200 \mathrm{~s}$ stress on the MCA in the control group, the NA group, and the EA group was decreased in comparison with the values at $0 \mathrm{~s}$, by $3.7 \mathrm{kPa} 3,2.8 \mathrm{kPa}$, and 3.5 $\mathrm{kPa}$, respectively; the $7.200 \mathrm{~s}$ stress on the MCA in the EA group decreased more than in the NA group at $22.5 \mathrm{kPa}$ initial stress $(\mathrm{p}<0.05$; paired t-test).

Figure 1 shows that the stress in each group decreased rapidly within the first $600 \mathrm{~s}$, while the stress relaxation curves tended to be stable when 7.200s was reached; the stress relaxation curve of each group exhibited logarithmic changes.

\section{Creep curves for the $M C A$}

The creep curves with initial stress on the MCA of $16 \mathrm{kPa}$ in different groups are shown in Figure 3, and those with stress on the MCA of $22.5 \mathrm{kPa}$ are shown in Figure 4.

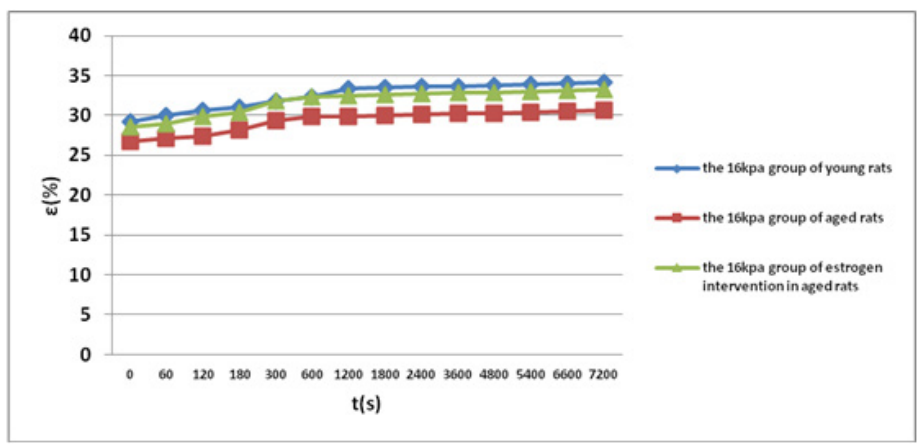

FIGURE 3 - Creep curves with initial stress of MCA of $16 \mathrm{Kpa}$ in different groups.

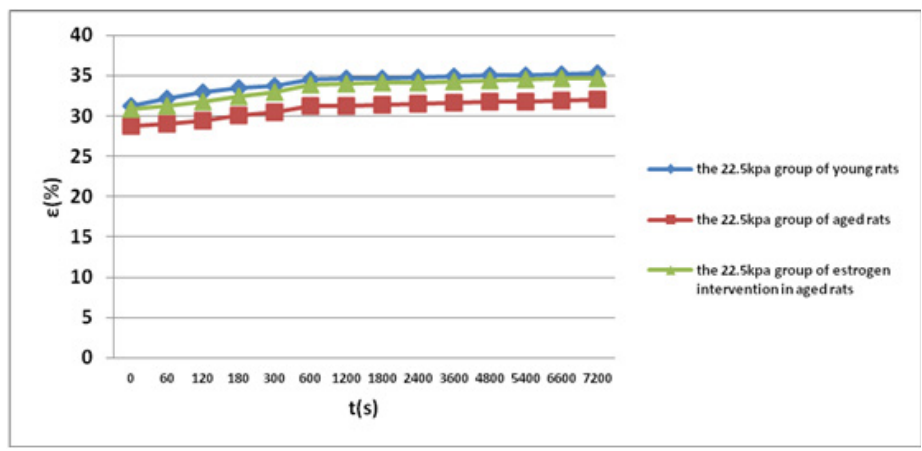

FIGURE 4 - Creep curves with initial stress of MCA of $22.5 \mathrm{Kpa}$ in different groups. 
When constant stress on the MCA was set at $16 \mathrm{kPa}$, the $7.200 \mathrm{~s}$ stress on the MCA in the control group, the NA group, and the EA group were increased in comparison with the values at $0 \mathrm{~s}$, by $4.9 \%, 3.8 \%$, and $4.6 \%(\mathrm{p}<0.05$; analysis of variance [ANOVA] and Scheffe's method), respectively, while the 7,200 s stress on the MCA in the EA group increased more than in the NA group $(p<0.05$; paired t-test). When the constant stress on the MCA was set at $22.5 \mathrm{kPa}$, the $7.200 \mathrm{~s}$ stress on the MCA in the control group, the NA group, and the EA group increased in comparison with the values at $0 \mathrm{~s}$, by $4.1 \%, 3.8 \%$, and $3.9 \%(\mathrm{p}<0.05$; ANOVA and Scheffe's method), respectively; the 7.200 s stress on the MCA in the EA group increased more than in the NA group $(\mathrm{p}<0.05$; paired t-test).

The strain in each group increased rapidly within the first $600 \mathrm{~s}$, then slowed, and the creep curves tended to become stable at 7.200s; the creep curve for each group exhibited logarithmic changes, and the normalized creep function curve of each group also exhibited logarithmic changes.

\section{Discussion}

The results showed that the creep curve for each group exhibited logarithmic changes. Each group exhibited rapidly decreasing stress and rapidly increasing strain in the initial 1200 s, after which the stress decreased and the strain increased slowly; when 7.200 s was reached, the stress relaxation curve and the creep curve basically became stable. The deformation of the MCA did not increase in proportion to the increase in stress, while exhibiting slow logarithmic changes; this may play a protective role under conditions of both normal blood pressure and symptomatic emergent blood pressure elevation. This experiment demonstrated that changes in sex hormones influenced the elasticity characteristics of the MCA in animals; EA increased the in vivo estrogen levels in aged rats, thus restoring viscoelasticity, consistent with the desired results.

Fukunari et al. ${ }^{12}$ reported that the contraction of spirallyarranged smooth muscle fibers could increase arterial axial tension, while the contraction of toroidally-arranged smooth muscle fibers could decrease axial arterial tension. Once the functions of smooth muscles are lost, they may be replaced by collagen fibers, and the mechanical properties may be altered. Meng et al. ${ }^{13}$ reported that the structures of cerebrovascular elastic tissues play important roles in maintaining the tension and elasticity of the cerebral blood vessel wall. The analysis showed that aged rats would develop atherosclerosis, and the contents and arrangements of arterial elastic fibers, collagen fibers, and smooth muscle fibers were changed; thus, the properties of stress relaxation and sustained-stress creep in the MCA in aged rats change with time. Therefore, the amounts of decreased stress and increased creep in the MCA in the NA group at 7.200s were less than in the control group and EA group. Estrogen could increase the content of elastic fibers in the arterial wall and reduce the content of collagen fibers, thus producing direct effects on arterial remodeling ${ }^{14}$. Duckles and Krause ${ }^{15}$ showed that large doses of estrogen could promote rat cerebral microtubules to release cGMP and NO in the early stages of cerebral ischemia, thus dilating blood vessels and increasing cerebral blood flow. Momoi et al. ${ }^{7}$ found that estrogen could induce the microvascular system of gonadectomized rats to release NO, which was much more obvious in female rats. Estrogen regulated the release of NO, improved cerebral capillary quality, and maintained cerebral blood flow, thus preventing cerebral ischemia and cerebral artery atherosclerosis. Oxygen free radicals are produced by enzymatic and non-enzymatic systems, and can attack the polyunsaturated fatty acids in biological membranes and initiate lipid peroxidation, leading to the formation of lipid peroxide MDA and new oxygen free radicals. Oxygen free radicals not only induce cell damage through polyunsaturated fatty acids in biological membranes, but also cause cell damage through the decomposition of hydrogen peroxide products. High or low MDA reflects the level of lipid peroxidation in the body, and indirectly reflects the level of reactive oxygen species (ROS) level ${ }^{16,17}$. In vivo production of a large amount of ROS and lipid peroxidation would be accompanied by the inactivation and reduction of a large number of antioxidant defense enzymes ${ }^{18,19}$. Wang et al. ${ }^{9}$ showed that the serum levels of MDA in an aged NA group were significantly higher than in an EA group. Superoxide dismutase (SOD) is an active substance in the body that can eliminate harmful substances produced in the process of metabolism. SOD has a special anti-aging effect in the human body. This study showed that serum levels of MDA in the EA group were significantly higher than in the aged NA group. This indicated that E2 could reduce ROS and MDA, improve antioxidation ability, inhibit the formation of lipid peroxidation products, and reduce injury by oxygen free radicals and lipid oxidation products. It plays a protective role in rat MCA, and restores stress relaxation and creep properties in aged female rats.

In this study, the properties of stress relaxation and creep were restored in the EA group, indicating that E2 plays an important role in increasing arterial flexibility, i.e., compliance.

Wang et al. ${ }^{9}$ investigated the effects of EA on the biomechanical properties of the MCA in aged female rats using a 1D extension method; the results showed that the maximum stretch stress, maximum strain, and elastic limits of the MCA in 
aged rats after EA were greater than those in the NA group, and the differences were significant $(\mathrm{p}<0.05)$; therefore, the conclusion was that E2 administration could improve elasticity, strength, and compliance of the MCA in aged rats. In this experiment, stress relaxation and creep methods were used to determine viscoelastic properties in MCA samples in young rats, aged rats, and aged rats with EA; 1D methods used in the literature ${ }^{9}$ were applied to study the effects of EA on the biomechanical properties of the MCA in aged female rats; although the research methods were different, the results showed that the decreased amount of 7.200s stress and the increased amount of 7.200s strain in the MCA in aged rats after EA were greater than in the NA group in this study, showing good consistency with a prior report ${ }^{9}$.

In contrast with this study, a report by Wang et al. ${ }^{9}$ investigated mechanical properties of the MCA, such as elasticity and strength. The MCA is a viscoelastic material, and its inherent stress relaxation and creep properties exist in order to meet the needs of its physiological functions. This study investigated the effects of EA on the viscoelasticity of the MCA in aged female rats using stress relaxation and creep methods, to determine changes according to stress, strain, and time. This was more conducive to determining the biomechanical properties of the MCA. It was feasible to study EA in aged rats using indexes including stress relaxation and creep properties.

The artery has viscoelastic properties; elasticity has normally been used as an arterial compliance indicator, and therefore has certain limitations; it would be better to use viscoelastic properties to reflect and measure arterial compliance. This experiment determined variation patterns in MCA stress- and strain-time, as well as stress relaxation curves and creep curves, in 3 groups; the characteristics of this study differed from those of previous studies by obtaining variable patterns of MCA stress- and strain-time at $13.3 \mathrm{kPa}, 16.0 \mathrm{kPa}$, and $22.5 \mathrm{kPa}$, and therefore had greater clinical relevance. This study quantitatively clarified and comparatively analyzed the viscoelastic properties of the MCA in each group, and used MCA viscoelastic parameters and serum MDA indicators to determine the effects of E2 administration in aged female rats; therefore, it provided a viscoelastic basis for preventing cerebrovascular disease in elderly women. Hence, the design and methods of this experiment were innovative.

The methods of sampling, sample preservation, and preconditioning among the groups were consistent; as a result, the data were reliable. Because of individual differences and limited specimen samples, the experimental data showed some dispersion, but still provide reference values for use of EA to prevent cerebral arterial disease in elderly women.

\section{Conclusion}

Estradiol administration inhibited the formation of lipid peroxidation products and restored middle cerebral arterial viscoelasticity in aged female rats.

\section{References}

1. Liu F, Benashski SE, Xu Y, Siegel M, McCullough LD. Effects of chronic and acuteoestrogen replacement therapy in aged animals after experimentalstroke. J Neuroendocrinol. 2012 Feb;24(2):31930. doi: 10.1111/j.1365-2826.2011.02248.x.

2. Yu HJ, Fei J, Chen XS, Cai QY, Liu HL, Liu GD, Yao ZX. Progesterone attenuates neurological behavioral deficits of experimental autoimmune encephalomyelitis through remyelination with nucleus-sublocalized Olig1 protein. Neurosci Lett. 2010 May 26;476(1):42-5. doi: 10.1016/j.neulet.2010.03.079.

3. Rahme R, Zuccarello M, Kleindorfer D, Adeoye OM, Ringer AJ. Decompressive hemicraniectomy for malignant middle cerebral artery territory infarction: is life worth living? J Neurosurg. 2012 Oct;117(4):749-54. doi: 10.3171/2012.6.JNS111140.

4. Weil AG, Rahme R, Moumdjian R, Bouthillier A, Bojanowski MW. Quality of life following hemicraniectomy for malignant MCA territory infarction. Can J Neurol Sci. 2011 May;38(3):434-8. PMID: 21515502.

5. Lobo RA, Clarkson TB. Different mechanisms for benefit and risk of coronary heart disease and stroke in early postmenopausal women: a hypothetical explanation. Menopause. 2011 Feb;18(2):237-40. PMID: 21341399.

6. Won CK, Kim MO, Koh PO. Estrogen modulates Bcl-2 family proteins in ischemic brain injury. J Vet Med Sci. 2006 Mar;68(3):27780. PMID: 16598173.

7. Momoi H, Ikomi F, Ohhashi T. Estrogen-induced augmentation of endothelium-dependent nitric oxide-mediated vasodilation in isolated rat cerebral small arteries. Jpn J Physiol. 2003 Jun;53(3): 193203. PMID: 14529580.

8. Ibrahim J, McGee A, Graham D, et al. Sex-specific differences in cerebral arterial myogenic tone in hypertensive and normotensive rats. Am J Physiol Heart Circ Physiol. 2006 Mar;290(3):H1081-9. PMID: 16214843.

9. Wang SJ, Zhao SJ, Wang YS, Yu T, Luo M. Effects of estrogen intervention on the biomechanical characteristics of serum SOD, MDA, and middle cerebral artery in aged female rats. Clin Exp Obstet Gynecol. 2015;42(3):295-9. PMID: 26151996.

10. Peng C, Zhang Q, Yang Q, Zhu Q. Strain and stress variations in the human amniotic membrane and fresh corpse autologous sciatic nerve anastomosis in a model of sciatic nerve injury. Neural Regen Res. 2012 Aug 15;7(23):1779-85. doi: 10.3969/j.issn.16735374.2012.23.003.

11. Wang Y, Li ZW, Luo M, Li YJ, Zhang KQ. Biological conduits combining bone marrow mesenchymal stem cells and extracellular matrix to treat long-segment sciatic nerve defects. Neural Regen Res. 2015 Jun;10(6):965-71. doi: 10.4103/1673-5374.158362.

12. Fukunari H, Oshima M, Torii R, Watanabe H, Hisada L. Fluidstructure interaction finite element analysis of middle cerebral artery aneurysm. J Biomech. 2006;39:S364. doi: 10.1016/S00219290(06)84460-X.

13. Meng H, Wang Z, Hoi Y, Gao L, Metaxa E, Swartz DD, Kolega J. Complex hemodynamics at the apex of an arterial bifurcation induces vascular remodeling resembling cerebral aneurysm initiation. Stroke. 2007 Jun;38(6):1924-31. PMID: 17495215. 
14. Vitale C, Fini M, Speziale G, Chierchia S. Gender differences in the cardiovascular effects of sex hormones. Fundam Clin Pharmacol. 2010 Dec;24(6):675-85. doi: 10.1111/j.1472-8206.2010.00817.x.

15. Duckles SP, Krause DN. Cerebrovascular effects of oestrogen: multiplicity of action. Clin Exp Pharmacol Physiol. 2007 Aug;34(8):801-8. doi: 10.1111/j.1440-1681.2007.04683.x.

16. Stentz FB, Umpierrez GE, Cuervo R, Kitabchi AE. Proinflammatory cytokines, markers of cardiovascular risks, oxidative stress, and lipid peroxidation in patients with hyperglycemic crises. Diabetes. 2004 Aug;53(8):2079-86. PMID: 15277389.

17. Qian J, Jiang F, Wang B, Yu Y, Zhang X, Yin Z, Liu C. Ophiopogonin $\mathrm{D}$ prevents $\mathrm{H} 2 \mathrm{O} 2$-induced injury in primary human umbilical vein endothelial cells. J Ethnopharmacol. 2010 Mar 24;128(2):438-45. doi: 10.1016/j.jep.2010.01.031.

18. Dohare P, Varma S, Ray M. Curcuma oil modulates the nitric oxide system response to cerebral ischemia/reperfusion injury. Nitric Oxide. 2008 Aug;19(1):1-11. doi: 10.1016/j.niox.2008.04.020.

19. Gandhi C, Zalawadia R, Balaraman R. Nebivolol reduces experimentally induced warmrenal ischemiareperfusion injuryinrats. Ren Fail. 2008;30(9):921-30. doi: 10.1080/08860220802353900.

\section{Correspondence:}

Hang Zhao

Department of Neurosurgery

The Sino-Japanese Friendship Hospital of Jilin University

Changchun 130031 China

Phone: +8643185095142

Fax: +86 43185095142

hangzhaocn@163.com

Received: Jun 26, 2016

Review: Aug 22, 2016

Accepted: Sep 20, 2016

Conflict of interest: none

Financial source: none

${ }^{1}$ Research performed at Department of Neurosurgery, Sino-Japanese Friendship Hospital, Jilin University, Changchun, China. 